\title{
The Evolution of Groups and Group Galaxies to Intermediate Redshifts
}

\author{
Tesla E. Jeltema ${ }^{1}$ John S. Mulchaey ${ }^{1}$ Lori M. Lubin $^{2}$, \\ and Christopher D. Fassnacht ${ }^{2}$ \\ ${ }^{1}$ The Observatories of the Carnegie Institution of Washington \\ 813 Santa Barbara St., Pasadena, CA 91101 email: tesla@ociw.edu \\ ${ }^{2}$ Department of Physics, University of California at Davis, One Shields Ave., Davis, CA 95616
}

\section{Introduction}

We have undertaken a multiwavelength project to study the properties of X-ray selected groups at intermediate redshifts $(0.2<z<0.6)$. Mulchaey et al. (2006) presented the spectroscopic confirmation of nine groups. Jeltema et al. (2006) presented the $\mathrm{X}$-ray properties of these groups based on observations taken with XMM-Newton. These observations confirm that these systems contain luminous, diffuse gas with luminosities and temperatures consistent with the correlations seen among groups and clusters at low redshift. We have obtained deeper spectroscopic follow-up of seven groups with the Keck and Gemini telescopes. Along with the HST imaging, we study the galaxy populations in these groups, and our main results are summarized below.

\section{Summary}

Overall, the galaxy populations in X-ray luminous groups are similar to those in clusters at the same redshift; they have large fractions of early-type galaxies $\left(f_{e} \sim 70 \%\right)$ and small fractions of galaxies with significant star formation $\left(f_{O I I} \sim 30 \%\right)$. We do not observe a strong evolution in the galaxy populations from those seen in X-ray luminous groups at low-redshift, indicating that the large numbers of early-type, passive galaxies in X-ray emitting groups were largely in place by $z \sim 0.4$. Both $f_{e}$ and $f_{O I I}$ are correlated with radius but do not reach the field value out to $\sim r_{500}$. However, we find significant variation in the galaxy populations between groups with some groups having field-like populations. Comparisons between the morphological and spectral properties of group galaxies reveals both gas-less mergers and a population of passive spirals. As noted in Mulchaey et al.(2006), unlike low-redshift, X-ray emitting groups, in some of these groups the brightest galaxy (BGG) does not lie at the center of the potential, and in most of the groups which do have a central BGG, the BGG has multiple components. These groups appear to represent a range of evolutionary stages in the formation of the BGG. Some groups have relatively large central galaxy densities, and one group contains a string of seven bright galaxies within a radius of $200 h_{70}^{-1} \mathrm{kpc}$ which have a lower velocity dispersion than the rest of the system. None of the central galaxies, including those with multiple components, have significant [OII] emission. These observations support a scenario in which BGGs are formed relatively late through gas-poor mergers.

\section{References}

Mulchaey, J.S., Lubin, L.M., Fassnacht, C., Rosati, P., \& Jeltema, T.E. 2006, ApJ 646, 133. Jeltema, T.E., Mulchaey, J.S., Lubin, L.M., Rosati, P., \& Böhringer, H. 2006, ApJ 649, 649. 